\title{
Delivery of EZH2-shRNA with mPEG-PEI nanoparticles for the treatment of prostate cancer in vitro
}

\author{
YINXIA WU ${ }^{1 *}$, JUNJIE YU $^{2 *}$, YONGBIAO LIU $^{3 *}$, LIN YUAN $^{3}$, HANG YAN $^{3}$, JING JING $^{3}$ and GUOPING XU ${ }^{3}$ \\ Departments of ${ }^{1}$ Oncology and ${ }^{2}$ Urology, Clinical Medical College of Yangzhou University, Yangzhou, Jiangsu 225001; \\ ${ }^{3}$ Department of Oncology, The First Affiliated Hospital of Nanjing Medical University, Nanjing, Jiangsu 210029, P.R. China
}

Received December 6, 2013; Accepted March 18, 2014

DOI: $10.3892 /$ ijmm.2014.1724

\begin{abstract}
Small interfering RNA (siRNA) is a promising therapeutic approach for castration-resistant prostate cancer (PCa). For the clinical application of siRNA, it is vital to find a safe and efficient gene transfer vector. Nanotechnology can provide a crucial advantage in developing strategies for cancer management and treatment by helping to improve the safety and efficacy of new therapeutic delivery vehicles. In this study, we describe a novel nanoparticle (mPEG-PEI) as an efficient non-viral carrier and found that this copolymer displayed enhanced efficiency in the shRNA-mediated knockdown of target genes. The enhancer of zeste homolog 2 (EZH2) is often elevated in castration-resistant $\mathrm{PCa}$ and has been implicated in the progression of human PCa. Targeting EZH2 may have therapeutic efficacy for the treatment of metastatic, hormone-refractory PCa. mPEG-PEI binds plasmid DNA yielding nanoparticles and these complexes exhibit low cytotoxicity and high gene transfection efficiency. Taken together, mPEG-PEI may be a promising non-viral gene carrier for the delivery of EZH2 short hairpin (sh)RNA to PC3 cells for advanced PCa therapy.
\end{abstract}

\section{Introduction}

Prostate cancer (PCa) can be treated by the removal of testicular androgens; however, androgen depletion is frequently associated with the recurrence of malignant neoplasms, defined as castration-resistant prostate cancer (CRPC), which has a poor prognosis (1). The enhancer of zeste homolog 2 (EZH2) belongs to the catalytic subunit of polycomb repressive complex 2 (PRC2) and primarily functions by silenc transcription through the trimethylation of histone H3 lysine 27 (H3K27me3) (2). $\mathrm{EZH} 2$ is frequently elevated in many types of cancer, including

Correspondence to: Professor Yongbiao Liu, Department of Oncology, The First Affiliated Hospital of Nanjing Medical University, 300 Guangzhou Road, Nanjing, Jiangsu 210029, P.R. China

E-mail: oncologistlyb@163.com

*Contributed equally

Key words: enhancer of zeste homolog 2, mPEG-PEI, short hairpin RNA, prostate cancer
PCa (3). EZH2 overexpression has also been linked to PCa cell invasion and metastasis and poor survival $(4,5)$. The expression of EZH2 has been implicated in the progression of human PCa, particularly to lethal CRPC (6). A recent study reported that the blockade of EZH2 derepression during androgen deprivation therapy may represent an effective strategy for the treatment of androgen-refractory $\mathrm{PCa}(7)$. Xu et al (8) demonstrated that in patients with CRPC overexpressing EZH2, EZH2-stimulated solo genes are frequently upregulated, and their higher expression levels correlate with poor survival. Therefore, the suppression of EZH2 expression may be a novel strategy for the treatment of metastatic, hormone-refractory PCa.

Gene therapy is a promising strategy for the treatment of various genetic and acquired diseases. The use of small interfering RNA (siRNA) is a novel and promising therapeutic approach, as it can be used to suppress gene expression in a highly specific manner for the treatment of genetic diseases (9). Although highly attractive as a cancer therapeutic strategy, a number of physiological obstacles need to be overcome to successfully introduce RNAi-based therapies into clinical practice. Therefore, there is an urgent need to discover new treatment methods and to find more effective vectors which may improve the efficiency of target gene expression in order to achieve greater success with gene therapy.

Non-viral gene delivery systems can be mainly divided into lipids, polymers and nanomaterials, and have been developed for siRNA delivery $(10,11)$. Among these delivery systems, the polymer or lipid-based ones have shown much improvement in delivering therapeutic genes. However, complex chemical synthetic procedures and harsh chemical reactions limit their application (12). Nanoparticle vectors have been extensively investigated for siRNA delivery due to their ease of production, safety, lower immune response, target tissue and cell specificity, facilitatation of cellular entry, ease of chemical modification and their ability to transfer larger pDNA molecules (13). Polyethylenimine (PEI) is a highly efficient carrier for siRNA delivery; however, the high density of positive charge on the surface of PEI molecules results in severe cytotoxicity, which has become a limiting factor for the application of PEI (14). Polyethylene glycol (PEG) moieties are engrafted to PEI polymers with the aim of reducing cytotoxicity and enhancing stability (15).

In the present study, we used the non-viral cationic polymer vector mPEG-PEI nanoparticles as a carrier and rebuilt the 
shRNA plasmid; we evaluated the gene-silencing efficiency in PCa cells. The results substantiated that the mPEG-PEI/ EZH2-shRNA nanoparticle complexes effectively suppressed EZH2 mRNA expression, thus rendering these complexes as a promising candidate for the treatment of advanced PCa and providing the basis for future research in clinical translation.

\section{Materials and methods}

Cells and cell culture. The PC3 human prostate carcinoma cell line was obtained from the Shanghai Cell Bank, Chinese Academy of Sciences, Shanghai, China and maintained in RPMI-1640 culture medium supplemented with $10 \%$ fetal bovine serum (FBS) (Gibco), $100 \mathrm{U} / \mathrm{ml}$ penicillin and $100 \mathrm{mg} /$ $\mathrm{ml}$ streptomycin at $37^{\circ} \mathrm{C}$ in an atmosphere of $5 \% \mathrm{CO}_{2}$.

Synthesis of mPEG-PEI. mPEG-PEI was synthesized according to previously described methods (16-18). First, $7.9 \mathrm{~g}$ of mPEG-1900 and $1.6 \mathrm{ml}$ pyridine were dissolved in dichloromethane. p-Nitrophenoxycarbonyl chloride (p-NPC) (3.2 g) was dissolved in dichloromethane. The mPEG solution was then added to the p-NPC solution drop by drop and continuously stirred for $24 \mathrm{~h}$ at room temperature to complete the reaction. The mixture product was then precipitated with petroleum ether, frozen, filtrated and vacuum-dried. Subsequently, $1.03 \mathrm{~g}$ of the above product and $3.72 \mathrm{~g}$ PEI (MW 25,000) were added to $50 \mathrm{ml}$ anhydrous chloroform, and the mixture was continuously stirred for $48 \mathrm{~h}$ at room temperature. The sample was subsequently dialyzed against distilled water in a dialysis tube (MWCO, 3,500 kDa) for 3 days and lyophilized to obtain mPEG-PEI. mPEG-PEI was stored at $-20^{\circ} \mathrm{C}$ for further use.

Characterization of $m P E G-P E I$. To characterize the chemical composition and confirm that mPEG-PEI was successfully synthesized, ${ }^{1} \mathrm{H}$ nuclear magnetic resonance $\left({ }^{1} \mathrm{H}-\mathrm{NMR}\right)$ spectra were recorded using a Varian 400 spectrometer (Varian, Palo Alto, $\mathrm{CA}, \mathrm{USA}$ ) at $400 \mathrm{mHz}$ in $\mathrm{CDCl}_{3}$ with tetramethylsilane as an internal reference. The prepared nanoparticles were dispersed in deionized water and examined at various temperatures. All experimental groups were examined 3 times. The chemical shift was expressed in parts per million $(\delta)$ by using the proton peak of $\mathrm{H}_{2} \mathrm{O}$ (set as $4.7 \mathrm{pm}$ ) as an internal reference, as previously described (16).

Preparation of $m P E G-P E I / p D N A$ complexes. The plasmid pcDNA3.1/EGFP expression vector with ampicillin resistance and EGFP report was obtained from GenePharma (Shanghai, China). EZH2 short hairpin RNA (shRNA) sequences and the EZH2-shRNA negative control sequence were synthesized and cloned into the pcDNA3.1/EGFP expression vector. We manipulated the charge ratio between the amino groups of mPEG-PEI and the pcDNA3.1/EGFP/EZH2-shRNA (N/P) as 20. The appropriate volumes of mPEG-PEI and pcDNA3.1/EGFP/ EZH2-shRNA solutions were quickly mixed together (mPEGPEI/pDNA) and vortexed at the speed of 2,500 rpm for $30 \mathrm{sec}$ and then incubated at room temperature for $30 \mathrm{~min}$ prior to use.

Measurement of particle size and zeta potential of $M P E G$ PEI/pDNA complexes. The mPEG-PEI/pDNA complexes were prepared at different $\mathrm{N} / \mathrm{P}$ ratios (5-50) and then diluted in ultra-pure water. The particle size and zeta potential of the mPEG-PEI/pDNA complexes were examined using a ZetaPlus Instrument (Malvern Instruments, Ltd., Worcestershire, UK) with an angle of detection of $90^{\circ} \mathrm{C}$ at room temperature. The experiment was carried out using a Zetasizer $3000 \mathrm{HSa}$ particle sizer (Malvern Instruments, Ltd.) with an angle of detection of $90^{\circ} \mathrm{C}$ at room temperature.

3-(4,5-dimethylthiazol-2-yl)-2,5-diphenyltetrazolium bromide (MTT) cytotoxicity assays. The cytotoxicity associated with mPEG-PEI was determined by MTT assay according to a previously described method (19). The PC3 cells were plated with $100 \mu 1$ culture mediun in 96-well plates at a density of 5,000 cells/well. Different mPEG-PEI concentrations and different N/P ratios were added to each well followed by incubation for $48 \mathrm{~h}$. MTT assay was performed and the absorbance of each well, which identifies the quantity of viable cells, was read at $590 \mathrm{~nm}$ on a microplate reader. Each assay was performed in triplicate with 3 independent replicates.

Gel retardation assay. The attachment of the mPEG-PEI/ pDNA complexes was assessed using gel retardation assays. The mPEG-PEI/pDNA complexes formed at various N/P ratios $(0,1,2,3,5,10$ and 20$)$ and was placed on $1 \%$ agarose gels containing $0.5 \mathrm{mg} / \mathrm{ml}$ ethidium bromide in $1 \mathrm{X}$ TBE buffer. mPEG-PEI/pDNA binding was analyzed by gel electrophoresis at $80 \mathrm{~V}$ for $40 \mathrm{~min}$. DNA bands in the gel imaging system were observed and photographed.

Hemagglutination assay. The agglutinating activity of mPEG-PEI/pDNA at different N/P ratios was examined in a 24-well plate. Briefly, fresh mouse blood was centrifuged at $2,000 \mathrm{rpm}$ for $20 \mathrm{~min}$ and the plasma and the buffy coat were discarded. Erythrocytes were washed 3 times by centrifugation at 2,000 rpm and were diluted in phosphate-buffered saline (PBS) to a final concentration of $1 \%(\mathrm{v} / \mathrm{v})$. Various N/P ratios were added to the erythrocyte suspension at ratio of 10 , 20 and 50 in a 24-well plate and incubated for $15 \mathrm{~min}$ at room temperature. The sample was placed on a microscope slide and hemagglutination was observed under an optical microscope.

In vitro transfection experiments. The day prior to transfection, the PC3 cells were seeded into a 24-well plate at density of $0.5 \times 10^{5}$ cells/well. mPEG-PEI/pDNA transfection complexes with different N/P ratios $(3,5,10$ and 20) were prepared and added into the well in a dropwise manner. After $6 \mathrm{~h}$ of incubation, the medium was replaced with fresh complete medium and the cells were incubated for an additional $48 \mathrm{~h}$ prior to analysis. EGFP expression was examined and photographed under a fluorescent microscope (Olympus Axiovert S100; Olympus, Tokyo, Japan) after an additional $48 \mathrm{~h}$ of incubation. The number of EGFP-positive cells and total cells in 5 randomly selected sections was counted, and the transfection efficiency was calculated by the ratio between the EGFP-positive cells and the total number of cells, as previously described (16).

Quantitative reverse transcription PCR ( $q R T-P C R)$. Total RNA was isolated from the PC3 cells using TRIzol reagent (Invitrogen, Carlsbad, CA, USA) according to the manufacturer's instructions. Reverse transcription and quantitative PCR 


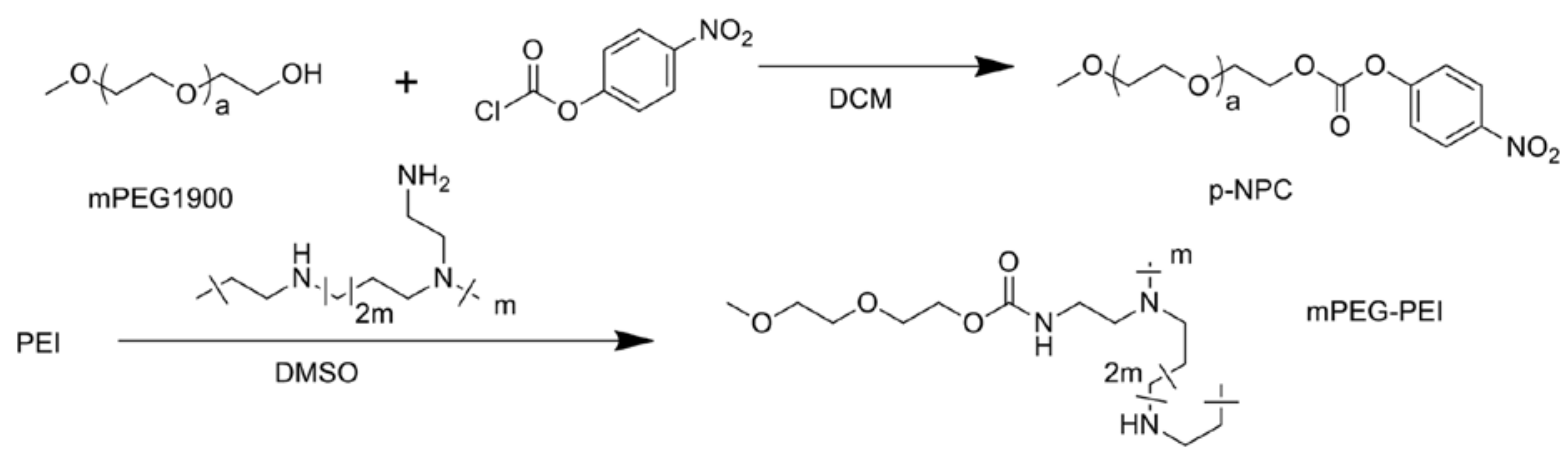

Figure 1. Schematic illustration of the synthetic process of mPEG-PEI. mPEG-PEI was synthesized through PEG moieties engrafted to the PEI polymers. PEG, polyethylene glycol; PEI, polyethylenimine.

were performed using the PrimeScript Reverse Transcription system and the SYBR Premix Ex Taq ${ }^{\mathrm{TM}}$ II kit (Takara, Dalian, China) according to the manufacturer's instructions. The relative mRNA expression compared to that of glyceraldehyde 3-phosphate dehydrogenase (GAPDH) was calculated using the $2^{-\Delta \mathrm{Ct}}$ method. The primers used were as follows: EZH2 forward, 5'-TGCAGTTGCTTCAGTACCCATAAT-3' and reverse, 5'-ATC CCCGTGTACTTTCCCATCATAAT-3'; GAPDH forward, 5'-TCGACAGTCAGCCGCATCTTCTTT-3' and reverse, 5'-ACCAAATCCGTTGACTCCGACCTT-3'. The quantitative PCR reactions were performed under the following conditions: $95^{\circ} \mathrm{C}$ for $2 \mathrm{~min}$, followed by 40 cycles of $95^{\circ} \mathrm{C}$ for $15 \mathrm{sec}$, and $60^{\circ} \mathrm{C}$ for $1 \mathrm{~min}$.

Western blot analysis. Protein samples were prepared by lysing the cells in modified RIPA buffer and protease inhibitor [phenylmethylsulfonyl fluoride (PMSF); Beyotime, Jiangsu, China]. Cell lysates were subjected to $10 \%$ SDS-polyacrylamide gel electrophoresis followed by electroblotting onto PVDF membranes. After blocking in 5\% skim milk, the membranes were probed with the specific primary antibody (anti-EZH2 or anti-GAPDH; Cell Signaling Technology, Beverly, MA, USA) and then incubated with a HRP-conjugated secondary antibody. Signals were visualized using the ECL chemiluminescence kit (Boster, Wuhan, China) and exposured to X-ray films.

Statistical analysis. Statistical analysis was performed using SPSS software version 17.0. The results are presented as the means \pm standard deviation (SD). The Student's t-test was used to assess statistically significant differences. A value of $\mathrm{P}<0.05$ was considered to indicate a statistically significant difference. All experiments were performed in triplicate.

\section{Results}

Synthesis and characterization of $m P E G-P E I$. The reaction scheme of mPEG-PEI is shown in Fig. 1. mPEG-PEI was prepared by the conjugation of $\mathrm{mPEG}-1900$ to PEI. The molecular structure of mPEG-PEI was characterized by measuring ${ }^{1} \mathrm{H}-\mathrm{NMR}$ in deuterium oxide. Fig. 2 illustrates that the proton peak appearing at 2.5-3.0 ppm was attributed to $-\mathrm{CH}_{2} \mathrm{CH}_{2} \mathrm{NH}$ - in PEI. The peaks at $3.6 \mathrm{ppm}$ were assigned to the protons of $-\mathrm{CH}_{2} \mathrm{CH}_{2} \mathrm{O}$ - in PEG. The peak at 7.25 was the chemical shifts of protons from solvent $\mathrm{D}_{2} \mathrm{O}$. This copolymer has both the characteristic absorption peaks of the proton in

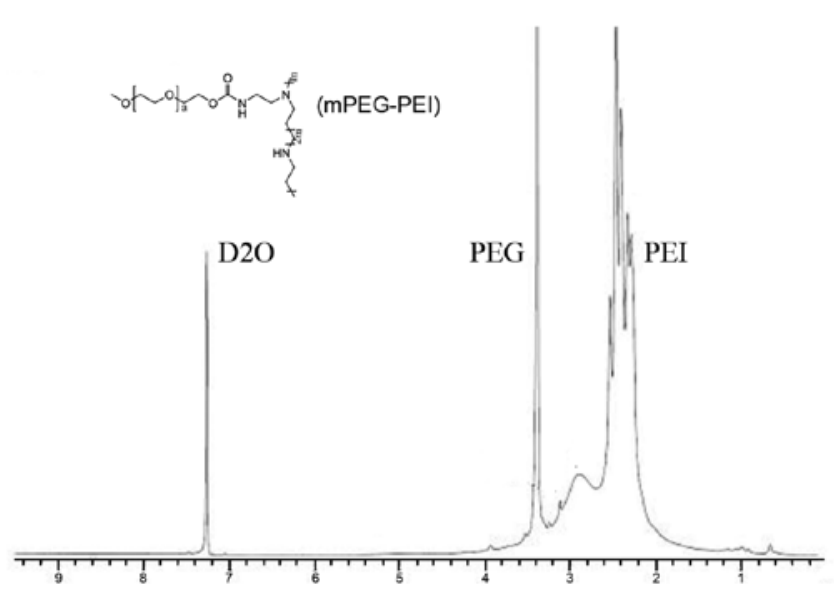

Figure 2. Representative ${ }^{1} \mathrm{H}$ nuclear magnetic resonance $\left({ }^{1} \mathrm{H}-\mathrm{NMR}\right)$ spectra of mPEG-PEI. The proton peak appearing at 2.5-3.0 ppm was attributed to $-\mathrm{CH}_{2} \mathrm{CH}_{2} \mathrm{NH}$ - in PEI. The peaks at $3.6 \mathrm{ppm}$ were assigned to the protons of $-\mathrm{CH}_{2} \mathrm{CH}_{2} \mathrm{O}$ - in PEG. The peak at 7.25 was a chemical shift of the protons from solvent $\mathrm{D}_{2} \mathrm{O}$. PEG, polyethylene glycol; PEI, polyethylenimine.

PEG and characteristic absorption peak in PEI, and it can be identified as PEG copolymer with PEI. These results indicated that mPEG-PEI was successfully synthesized.

Particle size and zeta potential characterization. The mPEG-PEI/pDNA complexes were evaluated for particle size and zeta potential. The particle size and zeta potential of the mPEG-PEI/pDNA complexes varied at the different N/P rations (Fig. 3). Dynamic light scattering (DLS) experiments using different mPEG-PEI/pDNA (N/P) ratios revealed that the particle size of the mPEG-PEI/DNA complexes decreased with the increasing N/P ratios. At an N/P ratio of 5, the particle size was approximately $183 \mathrm{~nm}$. The particle size reached approximately $90 \mathrm{~nm}$ at an N/P ratio of 10 , and then remained relatively constant between 70 and $90 \mathrm{~nm}$. When the N/P ratio increased, the size of the PEG-PEI/EZH2-shRNA complexes decreased, but the zeta potential increased. The zeta potential increased with the increased N/P ratio, but the particle size of the $\mathrm{mPEG}$ $\mathrm{PEI} / \mathrm{pDNA}$ complexes decreased.

Agarose gel retardation assay. An agarose gel retardation assay was performed to measure the ability of mPEG-PEI to bind DNA. mPEG-PEI/pDNA complexes formed at various $\mathrm{N} / \mathrm{P}$ ratios in a gel retardation assay (Fig. 4). This phenom- 


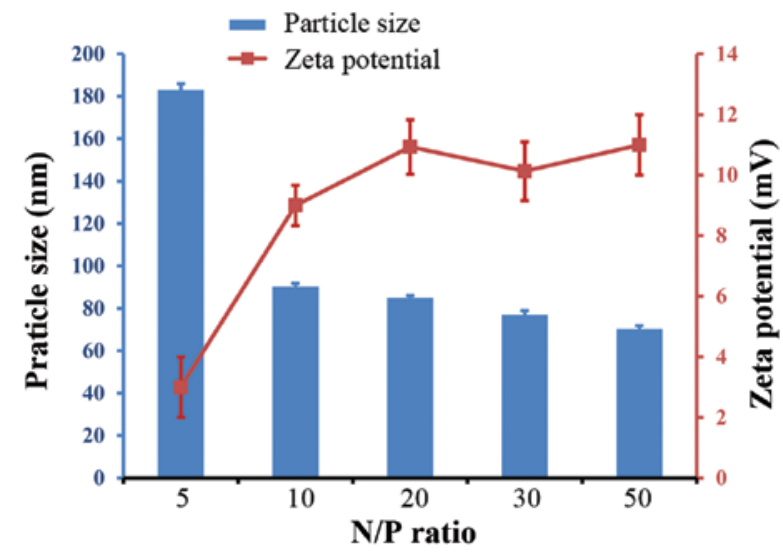

Figure 3. Particle size and zeta potential of the mPEG-PEI/pDNA complexes. Effective diameter and zeta potential of the mPEG-PEI/pDNA complexes at various $\mathrm{N} / \mathrm{P}$ ratios $(5,10,20,30$ and 50$)$. Zeta potentials were determined with an incident laser beam of $633 \mathrm{~nm}$ at a scattering angle of $90^{\circ}$ using a Malvern Zetasizer 3000 HSa. Data are the means \pm standard deviation (SD) $(\mathrm{n}=5)$. PEG, polyethylene glycol; PEI, polyethylenimine.

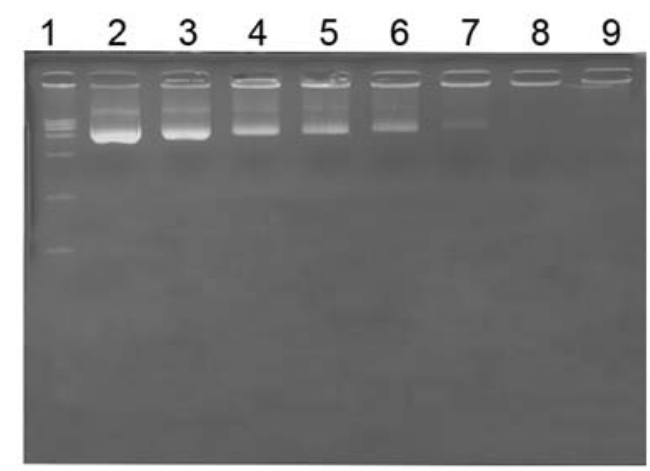

Figure 4. Agarose gel electrophoresis of the mPEG-PEI/pDNA complexes at different N/P ratios. Agarose gel electrophoresis of the mPEG-PEI/pDNA complexes at various w/w ratios. Lane 1, marker; lane 2, naked pDNA; lanes 3-9, complexes at $\mathrm{N} / \mathrm{P}$ ratios of $0.1,0.5,1,3,5,10$ and 20 , respectively. PEG, polyethylene glycol; PEI, polyethylenimine.

enon can be explained by the fact that the positive charges of mPEG-PEI were able to neutralize the negative charges of the phosphate groups in EZH2-shRNA, thus retarding in gel. mPEG-PEI combine with pDNA, which hinder the EB insert double-stranded DNA. mPEG-PEI completely retarded the migration of DNA when the N/P ratio was 10 , indicating that the mPEG-PEI/pDNA complexes were fully formed at this $\mathrm{N} / \mathrm{P}$ ratio.

Cytotoxicity assay. PC 3 cells were used to determine the cytotoxic effects of the nanoparticles by MTT assay. Cytotoxicity was measured at various concentrations of the mPEG-PEI nanoparticles (Fig. 5A) and mPEG-PEI/pDNA ratios (N/P) from 0-60 (Fig. 5B). PC3 cell viability displayed a decreasing trend with the increasing mPEG-PEI polymer concentration; when the mPEG-PEI nanoparticle concentration was $>50 \mu \mathrm{g} /$ $\mathrm{ml}$, there was a significant increase in toxicity, and the cell death rate was $>50 \%$. The cytotoxicity of the mPEG-PEI/ pDNA complexes increased as the N/P ratio increased. The mPEG-PEI/pDNA complexes showed very small or negligible cytotoxicity when the N/P ratio was $<50$. Cell viability
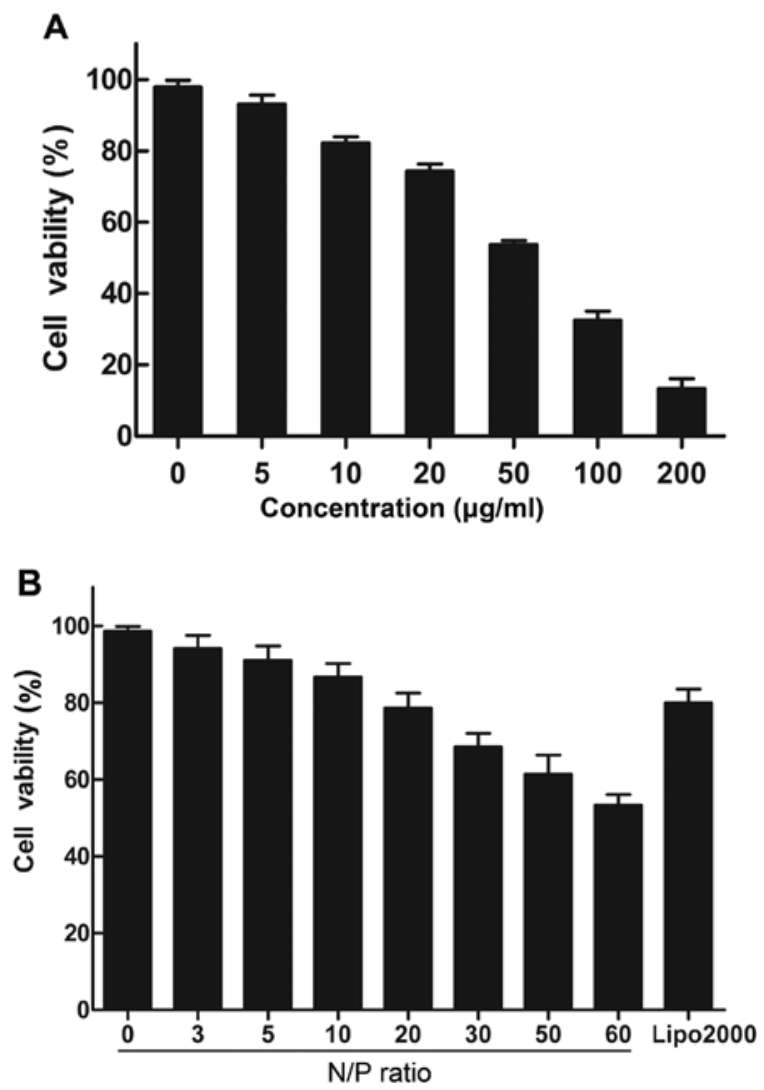

Figure 5. Cytotoxicity of mPEG-PEI. (A) Viability of PC3 cells exposed to nanoparticles with different exposure concentrations determined by MTT assay. Cells were treated with $0,5,10,20,50,100$ or $200 \mu \mathrm{g} / \mathrm{ml} \mathrm{mPEG-PEI.}$ (B) $\mathrm{PC} 3$ cells treated with mPEG-PEI/pDNA complexes at various N/P ratios from 0 to 60; Lipofectamine $^{\mathrm{TM}} 2000$ (Lipo2000) was used as a control. Results are the means \pm standard deviation (SD) of 3 independent experiments each carried out in triplicate. PEG, polyethylene glycol; PEI, polyethylenimine.

was $94.13 \pm 3.42$ and $78.6 \pm 3.94 \%$ at an N/P ratio of 3 and 20 , respectively. However, the cytotoxicity of mPEG-PEI/pDNA markedly increased at an N/P ratio of $>50$.

Hemagglutination assay. Hemagglutination assay with mouse erythrocytes was performed to assess the agglutinating activity of mPEG-PEI/pDNA at different N/P ratios. Hemagglutination assay for N/P ratios of 10, 20 and 50 is shown in Fig. 6. When the N/P ratio was 50, the mouse erythrocytes began to aggregate, whereas no agglutinating ability was observed at an $\mathrm{N} / \mathrm{P}$ ratio of 10 and 20 . These findings indicate that different $\mathrm{N} / \mathrm{P}$ ratios have different hemagglutination abilities. This is a significantl factor in determining which ratio is suitable for use in clinical applications.

Transfection efficiencyassay in vitro. The transfection efficiency of mPEG-PEI/pDNA at various N/P ratios was observed $48 \mathrm{~h}$ after transfection. Fluorescence images in the cells were evaluated under a fluorescence microscope (Fig. 7A). We compared the transfection efficiency of mPEG-PEI/pDNA with the transfection reagent, Lipofectamine ${ }^{\mathrm{TM}}$ 2000. The gene transfection efficiency correspondingly increased with the increasing N/P ratio from 3 to 20 , and the transfection efficiencies increased from $12.10 \pm 1.61$ to $64.67 \pm 3.92 \%$. The transfection efficiency of Lipofectamine $^{\mathrm{TM}} 2000$ was $62.63 \pm 3.62 \%$. The highest transfec- 


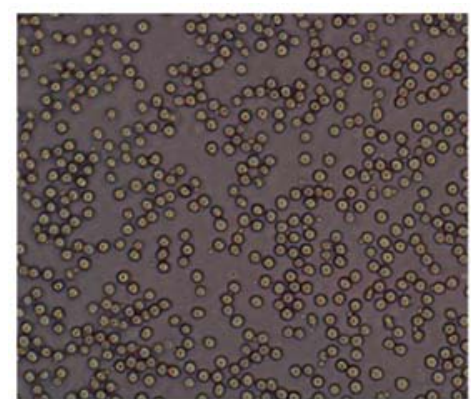

$\mathbf{N} / \mathbf{P}=\mathbf{1 0}$

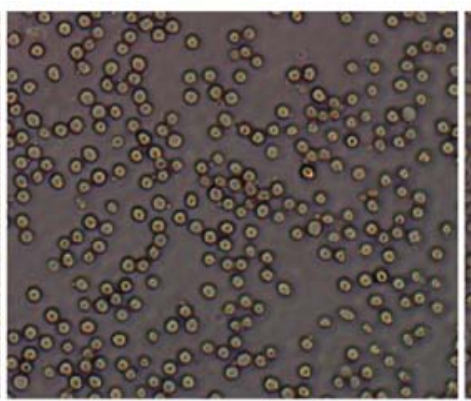

$\mathbf{N} / \mathbf{P}=\mathbf{2 0}$

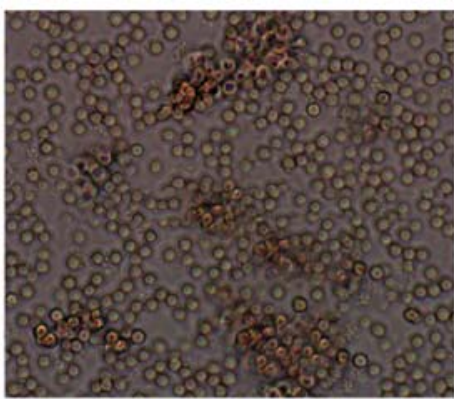

$\mathbf{N} / \mathbf{P}=\mathbf{5 0}$

Figure 6. Hemagglutination assay. Hemagglutination assay was performed to assess the agglutinating activity of mPEG-PEI/pDNA at different N/P ratios. Erythrocytes became scattered at an N/P ratio of 10 and 20. Erythrocytes began to aggregate with the mPEG-PEI/pDNA complexes at an N/P ratio of 50 .

A

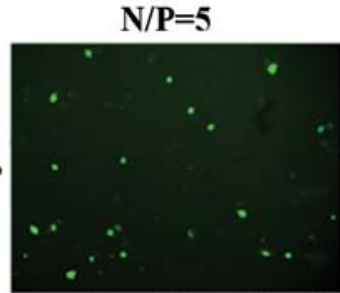

DIC
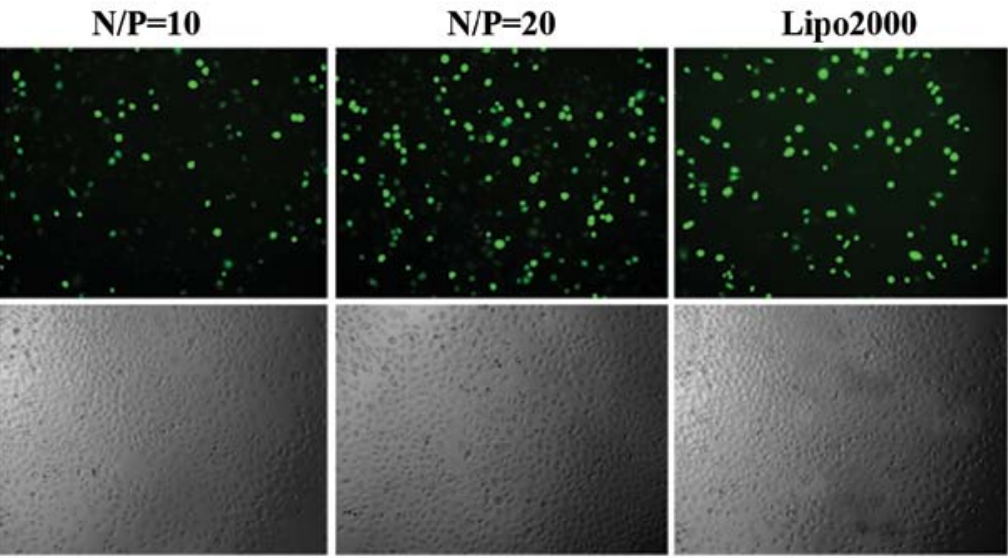

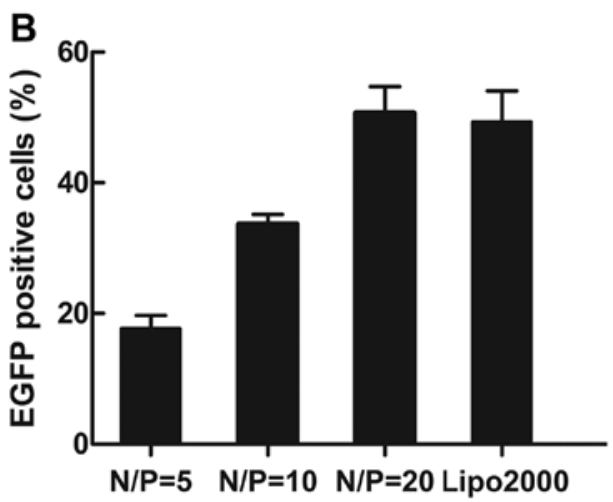

Figure 7. Gene transfection efficiency of mPEG-PEI. (A) Representative microscopic images of EGFP expression in PC3 cells at at different N/P ratios and Lipofectamine 2000 (Lipo2000; control) after 48 h of transfection. (B) Quantitative analysis of transfection efficiency of mPEG-PEI/pDNA complexes. Percentage of EGFP-positive cells per total amount of PC3 cells at indicated ratios (N/P) after $48 \mathrm{~h}$ of transfection [means \pm standard deviation $(\mathrm{SD}) \mathrm{n}=3$ ] PEG, polyethylene glycol; PEI, polyethylenimine.

tion activity of mPEG-PEI/pDNA was obtained at an N/P ratio of 20 in the PC3 cells (Fig. 7B).

Gene silencing effect of EZH2-shRNA. To determine whether the mPEG-PEI/pDNA particles knockdown EZH2 mRNA expression in vitro, we investigated the efficacy of the $\mathrm{mPEG}$ PEI/pDNA particles in the PC3 cells. mPEG-PEI/pDNA at an $\mathrm{N} / \mathrm{P}$ ratio of 20 was selected for the transfection of the PC3 cells. Following $48 \mathrm{~h}$ of treatment, EZH2 gene expression was measured by qRT-RCR and western blot analysis. As shown in Fig. 8A, the EZH2 mRNA expression was knocked down to $24.0 \pm 2.64$ and $28.47 \pm 2.90 \%$ by the $\mathrm{mPEG}-\mathrm{PEI} / \mathrm{pDNA}$ particles and Lipofectamine ${ }^{\mathrm{TM}}$ 2000/shRNA-EZH2 complex, respectively. The blank control and $\mathrm{mPEG}-\mathrm{PEI} / \mathrm{shRNA}$ control particle treatment groups showed no obvious silencing effect on the expression of EZH2. The cells transfected with the mPEG-PEI/pDNA particles showed a decreased EZH2 protein expression compared to the cells transfected with the negative control (Fig. 8B).

\section{Discussion}

$\mathrm{PCa}$ is the most common malignant disease and the second leading cause of cancer-related mortality in males in the 

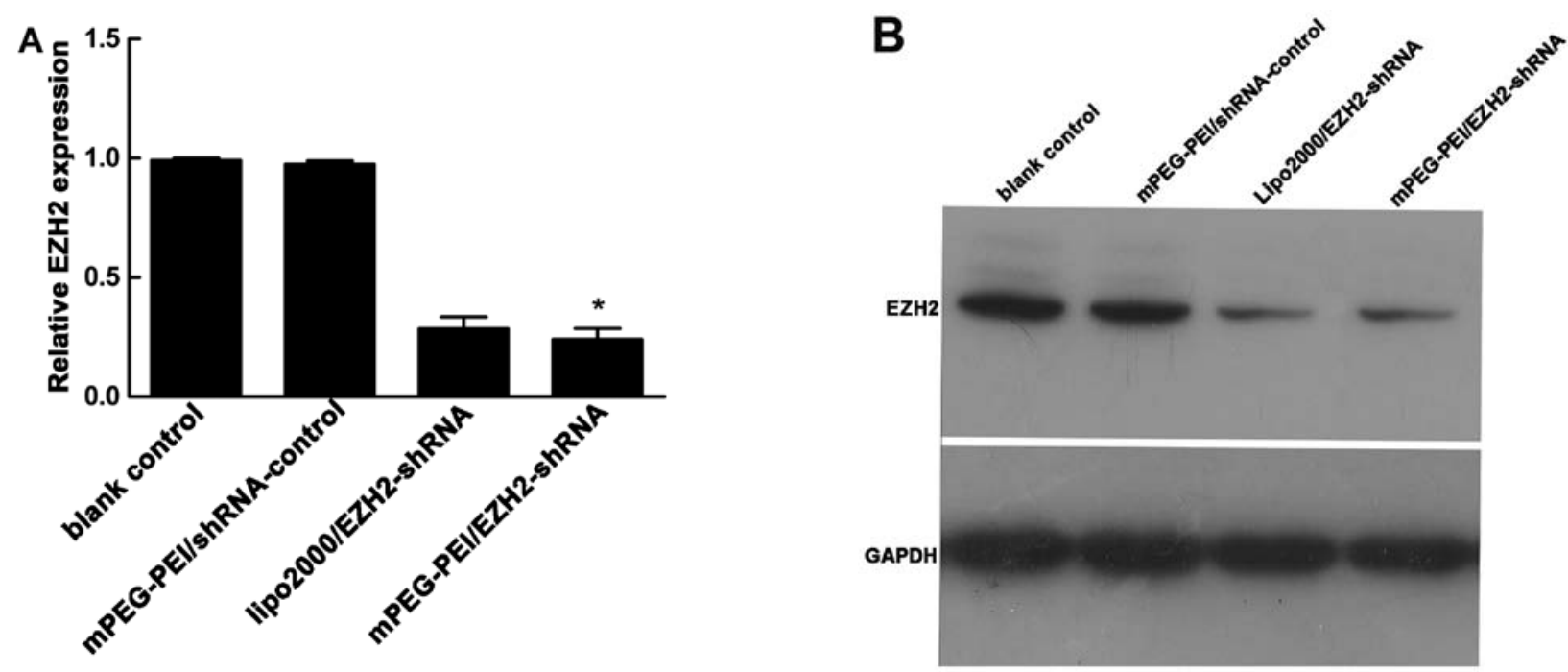

Figure 8. Gene silencing effect of mPEG-PEI/pDNA (N/P=20) in PC3 cell lines. (A) qRT-PCR for the analysis of EZH2 expression. (B) Western blot analysis of EZH 2 expression. All values are the means \pm standard deviation (SD) of 3 experiments ( $\mathrm{P}<0.05$ vs. blank control).

Western world (20). The majority of patients with PCa initially respond to androgen ablation, but eventually progress to CRPC, which is a lethal form of $\mathrm{PCa}$ that is an aggressive malignancy with a poor prognosis (21). Thus, it is crucial to discover an novel effective approaches for the treatment of CRPC.

It is mandatory to discover a novel and effective therapeutic strategy for CRPC. The EZH2 gene, which has been implicated in metastatic $\mathrm{PCa}$, belongs to an important component of PRC2 and regulates epigenetic gene silencing (22-24). Numerous studies have indicated that EZH2 is overexpressed in hormone-refractory, metastatic PCa $(5,6,25)$. The downregulation of the expression of EZH2 decreases prostate cell proliferation and ectopically expressed EZH2 in prostate cells enhances the proliferation and invasion of PCa cells (26). Since EZH2 is overexpressed and acts as an oncogene in PCa, it has been suggested to be a therapeutic target for metastatic, hormone-refractory PCa $(8,27)$.

The silencing of gene expression by siRNA is rapidly becoming a powerful tool for genetic analysis and represents a potential strategy for therapeutic product development $(28,29)$. Despite the enormous therapeutic potential of siRNAs, their delivery remains problematic due to unfavorable biodistribution profiles and poor intracellular bioavailability (30). An increasing number of studies have indicated that nanoparticles have low cytotoxicity and high gene transfection efficiency and may thus be potentially applied as a new vector system for gene delivery $(31,32)$. In our study, the non-viral vehicle, mPEG-PEI, was applied in the siRNA plasmid delivery to PCa cells to overcome the obstacles involved with the application of siRNA. Studies have shown that the adequate size and positive potential of nanocomplexes are essential for cellular uptake $(33,34)$.

In this study, the affinity of the mPEG-PEI/pDNA complexes was evaluated by agarose gel electrophoresis. The plasmid DNA was completely retarded when the N/P ratios of the mPEG$\mathrm{PEI} / \mathrm{pDNA}$ complexes were 10 and the charge of shRNA was completely neutralized. Therefore, it may be considered that mPEG-PEI completely complexed with shRNA at N/P ratios of $\geq 10$.
The relative surface charges of the resulting complexes were quantified with zeta potential measurements (16). The particle size and zeta potential of the mPEG-PEI/pDNA complexes were dependent on the N/P ratio. We designed different $\mathrm{N} / \mathrm{P}$ ratios to discover which is the optimal N/P ratio of the nanoparticles for transfection. At N/P ratios of $\geq 10$, the size of the nanoparticles was approximately $90.33 \pm 1.53 \mathrm{~nm}$ and the zeta potential was $9.0 \pm 0.67 \mathrm{mV}$. This can be partly explained by the fact that the electrostatic interaction of mPEG-PEI with siRNA becomes stronger when the N/P ratio is enhanced (35). Therefore, mPEG-PEI/pDNA complexes with N/P ratio of 10 are suitable for transfection.

The cytotoxicity of the mPEG-PEI/pDNA complexes was analyzed by MTT assay. Cytotoxicity was induced by mPEGPEI in a concentration-dependent manner; these results are consistent with those of a previous study (36). When the N/P ratios were $>30$, the cytotoxicity of PEG-PEI markedly increased and the cell viability was $<70 \%$. The results of hemagglutination assay also indicated that the mouse erythrocytes began to agglutinate when the N/P ratio was 50 and was not suitable for application.

We constructed the plasmid pcDNA3.1/EGFP/EZH2shRNA expression vector. This construct carries a coding sequence for the EGFP protein, providing the extra feature of an EGFP marker for monitoring the cell distribution of the nanoparticles. The increased transfection efficiency of mPEG$\mathrm{PEI} / \mathrm{pDNA}$ was observed at higher N/P ratios, reaching the highest transfection efficiency at an N/P ratio of 20, which displayed the most bright fluorescent spots. The present study shows that mPEG-PEI/pDNA complexes at an N/P ratio of $<10$ cannot achieve excellent cell transfection efficacy.

Our results revealed that the mRNA and protein levels of EZH2 were downregulated in the cancer tissues following transfection with mPEG-PEI/pDNA for $48 \mathrm{~h}$, indicating that mPEG-PEI can deliver EZH2-specific shRNA into PC3 cells, resulting in the inhibition of EZH2 expression.

In this study, we developed a novel strategy of nanoparticles loaded with EZH2-shRNA constructs for PCa gene therapy. 
mPEG-PEI/pDNA complexes were successfully synthesized with suitable physicochemical properties. More importantly, the mPEG-PEI/pDNA complexes exhibited low cytotoxicity and high tranfection efficiency. In addition, mPEG-PEI delivered EZH2-shRNA, effectively inhibiting the expression of EZH2 in the PC3 cells, which was a key target for the treatment of CRPC. At present, there are limited therapeutic options for CRPC. Therefore, mPEG-PEI may be a promising non-viral vector for delivering EZH2-shRNA plasmids to $\mathrm{PCa}$ cells and its application for CRPC therapy in vivo requires further investigation.

\section{References}

1. Cavalli G: Molecular biology. EZH2 goes solo. Science 338: 1430-1431, 2012

2. Cao Q, Mani RS, Ateeq B, et al: Coordinated regulation of polycomb group complexes through microRNAs in cancer. Cancer Cell 20: 187-199, 2011.

3. van Leenders GJ, Dukers D, Hessels D, et al: Polycomb-group oncogenes EZH2, BMI1, and RING1 are overexpressed in prostate cancer with adverse pathologic and clinical features. Eur Urol 52: 455-463, 2007.

4. Ren G, Baritaki S, Marathe H, et al: Polycomb protein EZH2 regulates tumor invasion via the transcriptional repression of the metastasis suppressor RKIP in breast and prostate cancer. Cancer Res 72: 3091-3104, 2012.

5. Karanikolas BD, Figueiredo ML and Wu L: Polycomb group protein enhancer of zeste 2 is an oncogene that promotes the neoplastic transformation of a benign prostatic epithelial cell line. Mol Cancer Res 7: 1456-1465, 2009.

6. Varambally S, Dhanasekaran SM, Zhou M, et al: The polycomb group protein EZH 2 is involved in progression of prostate cancer. Nature 419: 624-629, 2002.

7. Bohrer LR, Chen S, Hallstrom TC and Huang H: Androgens suppress EZH2 expression via retinoblastoma (RB) and p130-dependent pathways: a potential mechanism of androgenrefractory progression of prostate cancer. Endocrinology 151 $5136-5145,2010$.

8. $\mathrm{Xu} \mathrm{K}, \mathrm{Wu} \mathrm{ZJ}$, Groner AC, et al: EZH2 oncogenic activity in castration-resistant prostate cancer cells is Polycombindependent. Science 338: 1465-1469, 2012.

9. Pecot CV, Calin GA, Coleman RL, Lopez-Berestein G and Sood AK: RNA interference in the clinic: challenges and future directions. Nat Rev Cancer 11: 59-67, 2011.

10. Park TG, Jeong JH and Kim SW: Current status of polymeric gene delivery systems. Adv Drug Deliv Rev 58: 467-486, 2006.

11. Jing GJ, Fu ZG, Dan B, Lin LR, Yang TC and Shi SL: Development and evaluation of a novel nano-scale vector for siRNA. J Cell Biochem 111: 881-888, 2010.

12. Wang Z, Liu G, Zheng H and Chen X: Rigid nanoparticle-based delivery of anti-cancer siRNA: Challenges and opportunities. Biotechnol Adv: Sep 5, S0734-9750(13)00154-7, 2013 (Epub ahead of print).

13. Mintzer MA and Simanek EE: Nonviral vectors for gene delivery. Chem Rev 109: 259-302, 2009.

14. Huang W, Lv M and Gao Z: Polyethylenimine grafted with diblock copolymers of polyethylene glycol and polycaprolactone as siRNA delivery vector. J Control Release 152 (Suppl 1): e143-e145, 2011

15. Weber ND, Merkel OM, Kissel T and Muñoz-Fernández MA: PEGylated poly(ethylene imine) copolymer-delivered siRNA inhibits HIV replication in vitro. J Control Release 157: 55-63, 2012.

16. Xu Z, Jin J, Siu LK, et al: Folic acid conjugated mPEG-PEI600 as an efficient non-viral vector for targeted nucleic acid delivery. Int J Pharm 426: 182-192, 2012.
17. Veiseh $\mathrm{O}$, Kievit FM, Mok H, et al: Cell transcytosing poly-arginine coated magnetic nanovector for safe and effective siRNA delivery. Biomaterials 32: 5717-5725, 2011.

18. Shi S, Zhu X, Guo Q, et al: Self-assembled mPEG-PCL-g-PEI micelles for simultaneous codelivery of chemotherapeutic drugs and DNA: synthesis and characterization in vitro. Int J Nanomedicine 7: 1749-1759, 2012.

19. Liu Y, Liu Z, Wang Y, et al: Investigation of the performance of PEG-PEI/ROCK-II-siRNA complexes for Alzheimer's disease in vitro. Brain Res 1490: 43-51, 2013.

20. Jemal A, Siegel R, Ward E, Murray T, Xu J and Thun MJ: Cancer statistics, 2007. CA Cancer J Clin 57: 43-66, 2007.

21. Kirby M, Hirst C and Crawford ED: Characterising the castration-resistant prostate cancer population: a systematic review. Int J Clin Pract 65: 1180-1192, 2011.

22. Min J, Zaslavsky A, Fedele G, et al: An oncogene-tumor suppressor cascade drives metastatic prostate cancer by coordinately activating Ras and nuclear factor-kappaB. Nat Med 16: 286-294, 2010.

23. Tan J, Yang X, Zhuang L, et al: Pharmacologic disruption of Polycomb-repressive complex 2-mediated gene repression selectively induces apoptosis in cancer cells. Genes Dev 21: 1050-1063, 2007.

24. Cao R, Wang L, Wang H, et al: Role of histone H3 lysine 27 methylation in Polycomb-group silencing. Science 298: 1039-1043, 2002.

25. Yu J, Yu J, Rhodes DR, Tomlins SA, et al: A polycomb repression signature in metastatic prostate cancer predicts cancer outcome. Cancer Res 67: 10657-10663, 2007.

26. Bryant RJ, Cross NA, Eaton CL, Hamdy FC and Cunliffe VT: EZH2 promotes proliferation and invasiveness of prostate cancer cells. Prostate 67: 547-556, 2007.

27. Cao P, Deng Z, Wan M, et al: MicroRNA-101 negatively regulates Ezh2 and its expression is modulated by androgen receptor and HIF-1alpha/HIF-1beta. Mol Cancer 9: 108, 2010.

28. Takeshita F, Minakuchi Y, Nagahara S, et al: Efficient delivery of small interfering RNA to bone-metastatic tumors by using atelocollagen in vivo. Proc Natl Acad Sci USA 102: 12177-12182, 2005.

29. Monteagudo S, Pérez-Martínez FC, Pérez-Carrión MD, et al: Inhibition of p42 MAPK using a nonviral vector-delivered siRNA potentiates the anti-tumor effect of metformin in prostate cancer cells. Nanomedicine (Lond) 7: 493-506, 2012.

30. Giger EV, Castagner B, Räikkönen J, Mönkkönen J and Leroux JC: siRNA transfection with calcium phosphate nanoparticles stabilized with PEGylated chelators. Adv Healthc Mater 2: 134-144, 2013.

31. Li YH, Shi QS, Du J, et al: Targeted delivery of biodegradable nanoparticles with ultrasound-targeted microbubble destructionmediated hVEGF-siRNA transfection in human PC-3 cells in vitro. Int J Mol Med 31: 163-171, 2013.

32. Liu XQ, Xiong MH, Shu XT, Tang RZ and Wang J: Therapeutic delivery of siRNA silencing HIF-1 alpha with micellar nanoparticles inhibits hypoxic tumor growth. Mol Pharm 9: 2863-2874, 2012.

33. Wang Y, Su J, Cai W, et al: Hepatocyte-targeting gene transfer mediated by galactosylated poly(ethylene glycol)-graft-polyethylenimine derivative. Drug Des Devel Ther 7: 211-221, 2013.

34. Wu Y, Wang W, Chen Y, et al: The investigation of polymersiRNA nanoparticle for gene therapy of gastric cancer in vitro. Int J Nanomedicine 5: 129-136, 2010.

35. Aigner A: Delivery systems for the direct application of siRNAs to induce RNA interference (RNAi) in vivo. J Biomed Biotechnol 2006: 71659, 2006

36. Liang B, He ML, Xiao ZP, et al: Synthesis and characterization of folate-PEG-grafted-hyperbranched-PEI for tumor-targeted gene delivery. Biochem Biophys Res Commun 367: 874-880, 2008 . 